\title{
VARIAÇÃO RADIAL DAS PROPRIEDADES FÍSICAS DA MADEIRA DE ANGICO PRETO (Anadenanthera peregrina var. falcata) JOVEM
}

\section{PHYSICAL PROPERTIES RADIAL PROFILE OF THE ANGICO PRETO (Anadenanthera peregrina var. falcata) JUVENILE WOOD}

\author{
Maria Cecília Mota Docha ${ }^{1}$; Matheus De Oliveira Lopes²; Paulo Henrique Moreira³ ${ }^{3}$ Tamara \\ Kelly Marques Rocha Nunes ${ }^{4}$; Christovão Pereira Abrahão ${ }^{5}$
}

DOI: $\underline{\text { https://doi.org/10.31692/978-65-991061-7-0.345-350 }}$

\section{INTRODUÇÃO}

A Anadenanthera peregrina, popularmente conhecida como angico, é uma espécie pioneira a secundária inicial, comum na vegetação secundária, principalmente na fase de capoeirão. É encontrada naturalmente na Floresta Estacional Semidecidual Submontana (LORENZI, 2009). Estas espécies apresentam importância econômica e ecológica, sendo utilizadas na indústria moveleira, na construção civil e rural, na extração de taninos para utilização em curtumes e como fonte de combustível (lenha e carvão) (LORENZI, 2000). São, ainda, espécies recomendadas em sistemas agroflorestais e em projetos de reflorestamento de áreas degradadas por apresentarem rápido crescimento, eficiente fixação de nitrogênio e resistência a ventos fortes (LORENZI 2000;2009; PAES et al., 2006; FERRAZ et al., 2006).

Do ponto de vista tecnológico, tão importante quanto o estudo individual é o diagnóstico da variabilidade, que ocorre dentro da árvore, tanto no sentido transversal ou radial (medula-casca), como no sentido longitudinal (base-topo) (AGUIRRE et al., 2008). É relatado na literatura, dificuldades no uso da madeira em relação a sua qualidade, devido às proporções de madeira adulta e juvenil, ocasionando a diminuição na resistência e promovendo deformações durante e após o emprego da madeira serrada seca (RAMOS et al., 2011).

Pela importância e falta de estudos sobre as características tecnológicas da madeira desta espécie, o objetivo do trabalho é descrever os perfis radiais de variação para algumas

\footnotetext{
1 Programa de Pós-Graduação em Ciência Florestal, Universidade Federal dos Vales do Jequitinhonha e Mucuri, Departamento de Engenharia Florestal - Campus JK, Diamantina-MG, Brasil, (mcmdocha@gmail.com)

2 Programa de Pós-Graduação em Ciência Florestal, Universidade Federal dos Vales do Jequitinhonha e Mucuri, Departamento de Engenharia Florestal - Campus JK, Diamantina-MG, Brasil, (matheuoliveira@ gmail.com)

3 Programa de Pós-Graduação em Ciência Florestal, Universidade Federal dos Vales do Jequitinhonha e Mucuri, Departamento de Engenharia Florestal - Campus JK, Diamantina-MG, Brasil, (paulohengflo@gmail.com)

${ }^{4}$ Graduação em Engenharia Florestal, Universidade Federal dos Vales do Jequitinhonha e Mucuri, Departamento de Engenharia Florestal - Campus JK, Diamantina-MG, Brasil, (tamaraengf@gmail.com)

5 Prof. Dr. Ciência Florestal, Universidade Federal dos Vales do Jequitinhonha e Mucuri, Departamento de Engenharia Florestal - Campus JK, Diamantina-MG, Brasil, (cabrahao@gmail.com )
} 
propriedades físicas da madeira de angico preto (Anadenanthera peregrina).

\section{FUNDAMENTAÇÃO TEÓRICA}

A madeira juvenil ocorre tanto nas folhosas como nas coníferas e é aquela formada pelo câmbio nos primeiros anos de vida. Por ser mais jovem, esta região apresenta grandes diferenças anatômicas, químicas e físicas, que ocorrem no sentido da medula para a casca do tronco, se comparada com a madeira adulta, como: comprimento de fibra, ângulo fibrilar, proporções dos tipos de células, diâmetro celular, espessura da parede celular, teores de celulose, lignina e de umidade. Estas características fazem com que propriedades como massa específica, resistência mecânica e retratibilidade também variem. A baixa resistência mecânica, os empenamentos e as excessivas contrações longitudinais são problemas decorrentes da formação da madeira juvenil. (EVANS et al., 2000; ZOBEL; BUIJETNEN, 1989).

O conhecimento das propriedades de cada madeira é importante para melhor utilização. A massa específica, além de indicadora da qualidade da madeira, pode ser um importante subsídio para avaliação econômica da floresta, podendo, para fins práticos, tornar um indicativo do uso final da madeira (TRUGILHO et al., 1990).

\section{METODOLOGIA}

Foram obtidas seções radiais na altura do DAP (1,30 m em relação ao solo) de três árvores, cada disco com $5 \mathrm{~cm}$ de altura, retirados em um fragmento de mata secundária em região de transição entre Mata Atlântica e Cerrado, na fazenda Lagoa Dourada, no município de Águas Vermelhas - MG. A altura dos discos foi reduzida para $2 \mathrm{~cm}$, em serra de fita. Os diâmetros à altura do peito para as árvores 1,2 e 3 foram $12,5 \mathrm{~cm}, 6,4 \mathrm{~cm}$ e $5,4 \mathrm{~cm}$, respectivamente. Ainda, é importante atentar para o fato de que a Árvore 1 possuía uma bifurcação exatamente na altura onde foram retirados os discos.

Os discos foram lixados para facilitar a observação dos anéis de crescimento, utilizando lixas de granulometria de 36, 60 e 100 grãos, respectivamente. Após o lixamento foram registradas imagens da superfície dos discos com resolução de 600 dpi, através de um scanner de mesa para a documentação fotográfica que é útil na interpretação dos resultados dos perfis de densidade. Em seguida, foram determinados os menores e maiores diâmetros dos discos.

Foram retiradas as amostras a partir dos discos, com espessura média de $2 \mathrm{~mm}$, seccionando-as no sentido medula-casca. Foi utilizado para o seccionamento uma faca 
pressionada no sentido das fibras. Os discos das três árvores geraram 101 amostras que foram colocadas em um dessecador com água e aplicado a vácuo, para que fossem completamente saturados. Um dos discos apresentava bifurcação e dele foi retirado um número maior de amostras, que foram 47.

Com as amostras já saturadas, foi determinado peso saturado e o empuxo saturado, com o auxílio de uma balança analítica e um béquer contendo mercúrio líquido. Foram realizadas repetições para as determinações de volume e peso, com as amostras secas ao ar $\left(22{ }^{\circ} \mathrm{C}\right.$ e $50,3 \%$ de umidade relativa) e, depois, com as amostras secas em estufa com termocirculação de ar à $105^{\circ} \mathrm{C}$, até atingirem peso constante. Após, foi possível calcular os respectivos volumes, as densidades básicas, saturadas, ao ar e secas, a umidade saturada e a de equilíbrio higroscópico, além das contrações volumétricas ao ar e contrações volumétricas totais.

Para a comparação dos valores das propriedades de cada árvore, foi utilizado análise de variância e testes de médias com o nível de significância de $5 \%$, sendo utilizado o teste t de Student.

\section{RESULTADOS E DISCUSSÕES}

A contagem dos anéis de crescimento das árvores permitiu a estimativa da idade das árvores entre 10 e 13 anos.

A análise de variância detectou diferenças significativas para todas as propriedades, com exceção da umidade de equilíbrio higroscópico e da contração volumétrica total, quando comparadas as três árvores em estudo (Tabela 1).

Tabela 1. Resultados da análise de variância comparando as propriedades físicas das três árvores em estudo. Fonte: Própria. Legenda: D_Bas= Densidade básica; D_Sat= Densidade Saturada; D_Ar= Densidade seca ao ar;

D_Sec= Densidade seca em estufa; U_Sat= Umidade Saturada; UEH= Umidade de equilíbrio higroscópico;

C_Vol_Ar= Contração Volumétrica ao ar; C_Vol_Tot= Contração volumétrica total. Para as propriedades marcadas com “*” não foram detectadas diferenças significativas entre as árvores com $5 \%$ de significância, pelo teste $\mathrm{t}$.

\begin{tabular}{ccccccccc}
\hline & SQ & GL & QM & SQR & GLR & QMR & F & p \\
\hline D_Bas & 0,07 & 2 & 0,036 & 0,14 & 98 & 0,0015 & 24,89559 & 0,000000 \\
D_Sat & 0,01 & 2 & 0,005 & 0,08 & 98 & 0,0008 & 6,46086 & 0,002313 \\
D_Ar & 0,12 & 2 & 0,059 & 0,22 & 98 & 0,0023 & 26,08247 & 0,000000 \\
D_Sec & 0,09 & 2 & 0,046 & 0,21 & 98 & 0,0022 & 21,00330 & 0,000000 \\
U_Sat & 17312,12 & 2 & 8656,060 & 29713,29 & 98 & 303,1969 & 28,54931 & 0,000000 \\
UEH* & 1,51 & 2 & 0,754 & 39,17 & 98 & 0,3997 & 1,88719 & 0,156962 \\
C_Vol_Ar & 31,54 & 2 & 15,772 & 135,52 & 98 & 1,3828 & 11,40586 & 0,000035 \\
C_Vol_Tot* & 6,77 & 2 & 3,387 & 315,43 & 98 & 3,2186 & 1,05223 & 0,353068 \\
\hline
\end{tabular}

Na Tabela 1 percebe-se que as médias para a densidade básica, densidade da madeira seca ao ar, densidade da madeira seca em estufa, umidade de saturação e contração 
volumétrica ao ar apresentaram-se diferentes entre as três árvores estudadas, já as médias para umidade de equilíbrio higroscópico e contração volumétrica total não apresentaram diferença.

A Tabela 2 apresenta os valores médios, mínimos e máximos, bem como os desvios padrões para as propriedades estudadas. Para os valores de densidade da madeira saturada, as árvores 2 e 3 não apresentaram diferenças significativas entre si e as médias para contrações volumétricas ao ar, não apresentaram diferenças entre as árvores 1 e 3.

Tabela 2. Valores médios, mínimos, máximos e desvios padrões para as variáveis estudadas. Fonte: Própria.

Legenda: D_Bas= Densidade básica; D_Sat= Densidade Saturada; D_Ar= Densidade seca ao ar; D_Sec= Densidade seca em estufa; U_Sat= Umidade Saturada; UEH= Umidade de equilíbrio higroscópico; C_Vol_Ar= Contração Volumétrica ao ar; C_Vol_Tot= Contração volumétrica total. Médias na mesma linha, seguidas pela mesma letra, são iguais a 5\% de probabilidade pelo teste t.

\begin{tabular}{cccccccccc}
\hline Árvore & & D_Bas & D_Sat & D_Ar & D_Sec & U_Sat & UEH & C_Vol_Ar & C_Vol_Tot \\
\hline \multirow{6}{*}{$\mathbf{1}$} & Média & $\mathbf{0 , 4 9}$ A & $\mathbf{1 , 1 7} \mathbf{A}$ & $\mathbf{0 , 5 9} \mathbf{A}$ & $\mathbf{0 , 5 5} \mathbf{A}$ & $\mathbf{1 4 2 , 3 2} \mathbf{A}$ & $\mathbf{1 4 , 1 4} \mathbf{A}$ & $\mathbf{7 , 2 6}$ A & $\mathbf{1 3 , 2 7} \mathbf{A}$ \\
& Mínimo & 0,38 & 1,08 & 0,48 & 0,41 & 109,46 & 12,34 & 3,74 & 8,29 \\
& Máximo & 0,59 & 1,23 & 0,70 & 0,67 & 192,26 & 15,48 & 10,00 & 17,63 \\
& Des.Pad & 0,04 & 0,03 & 0,05 & 0,05 & 17,78 & 0,67 & 1,37 & 1,77 \\
\hline \multirow{6}{*}{$\mathbf{2}$} & Média & $\mathbf{0 , 4 2} \mathbf{B}$ & $\mathbf{1 , 1 5} \mathbf{B}$ & $\mathbf{0 , 5 1} \mathbf{B}$ & $\mathbf{0 , 4 8} \mathbf{B}$ & $\mathbf{1 7 3 , 2 4} \mathbf{B}$ & $\mathbf{1 3 , 9 4} \mathbf{A}$ & $\mathbf{8 , 6 0} \mathbf{B}$ & $\mathbf{1 3 , 7 5} \mathbf{A}$ \\
& Mínimo & 0,36 & 1,09 & 0,43 & 0,41 & 144,86 & 12,38 & 5,89 & 10,80 \\
& Máximo & 0,46 & 1,20 & 0,55 & 0,53 & 215,37 & 14,72 & 10,52 & 15,63 \\
& Des.Pad & 0,02 & 0,02 & 0,03 & 0,03 & 16,34 & 0,52 & 0,97 & 1,21 \\
\hline \multirow{6}{*}{$\mathbf{3}$} & Média & $\mathbf{0 , 4 5} \mathbf{C}$ & $\mathbf{1 , 1 5} \mathbf{B}$ & $\mathbf{0 , 5 4} \mathbf{C}$ & $\mathbf{0 , 5 1} \mathbf{C}$ & $\mathbf{1 5 9 , 2 7} \mathbf{C}$ & $\mathbf{1 4 , 2 8} \mathbf{A}$ & $\mathbf{7 , 6 4} \mathbf{A}$ & $\mathbf{1 3 , 8 2} \mathbf{A}$ \\
& Mínimo & 0,38 & 1,10 & 0,45 & 0,42 & 121,75 & 12,22 & 5,71 & 10,33 \\
& Máximo & 0,54 & 1,19 & 0,64 & 0,62 & 193,47 & 15,48 & 9,69 & 21,23 \\
& Des.Pad & 0,04 & 0,03 & 0,05 & 0,05 & 17,79 & 0,66 & 0,94 & 2,29 \\
\hline \multirow{6}{*}{ Geral } & Média & $\mathbf{0 , 4 6}$ & $\mathbf{1 , 1 6}$ & $\mathbf{0 , 5 5}$ & $\mathbf{0 , 5 2}$ & $\mathbf{1 5 4 , 7 9}$ & $\mathbf{1 4 , 1 2}$ & $\mathbf{7 , 7 1}$ & $\mathbf{1 3 , 5 3}$ \\
& Mínimo & 0,36 & 1,08 & 0,43 & 0,41 & 109,46 & 12,22 & 3,74 & 8,29 \\
& Máximo & 0,59 & 1,23 & 0,70 & 0,67 & 215,37 & 15,48 & 10,52 & 21,23 \\
& Des.Pad & 0,05 & 0,03 & 0,06 & 0,06 & 21,69 & 0,64 & 1,29 & 1,79 \\
\hline
\end{tabular}

Mori et al. (2003) relataram o valor de $0,64 \mathrm{~g} / \mathrm{cm}^{3}$ para a densidade básica da madeira de uma árvore de Anadenanthera peregrina. Teixeira (2008), por sua vez, relatou o valor de $0,66 \mathrm{~g} / \mathrm{cm}^{3}$ para a média de 7 árvore de Anadenanthera peregrina. A grande diferença entre os valores da literatura e aqueles mostrados na Tabela 2 deve-se a fatores genéticos e ambientais, além da idade das árvores, sendo as árvores que tiveram aqui determinadas suas propriedades possivelmente mais jovens que aquelas estudadas pelas autoras, dado o seu pequeno diâmetro médio. Já a variabilidade desta propriedade dentro de cada árvore e considerando-se os resultados das 3 árvores em conjunto, apresentou-se dentro do que é tido como esperado para uma espécie, com coeficiente de variação em torno de $10 \%$ (FPL, 2010).

Os valores para as contrações volumétricas totais $(13,53 \%)$ podem ser classificados como altos e estão acima do que Teixeira (2008) encontrou para Anadenanthera peregrina $(9,64 \%)$.

Já os valores para a umidade de equilíbrio higroscópico (UEH) apresentaram-se 
ligeiramente elevados para as condições ambientais em que foram determinados $\left(22^{\circ} \mathrm{C}\right.$ e 50,3\% de Umidade Relativa). Tal fato pode estar relacionado à presença de madeira de tração nas amostras, uma vez que as três árvores possuíam anomalias anatômicas causadas por esforços mecânicos, sugeridas pela presença da bifurcação na árvore 1 e pela excentricidade da medula em todas elas. Desta forma, as alterações químicas presentes na madeira de tração podem ter influenciado na sua higroscopicidade.

A madeira de angico, apresentou valores de densidade básica que podem ser classificados de médios a baixos. Entretanto, as árvores aqui avaliadas são jovens e esses valores podem crescer com a idade das árvores. A retratibilidade volumétrica mostrou-se um tanto elevada, o que suscita dúvidas acerca da utilidade desta madeira para usos mais nobres, como em movelaria.

O padrão de variação da densidade da madeira para esta espécie é do Tipo 1 (PANSHIN e DE ZEEW, 1970), com a tendência de crescimento em direção à casca. TEIXEIRA (2008) encontrou também uma tendência de crescimento radial da densidade básica da madeira de Anadenanthera peregrina.

\section{CONCLUSÕES}

O perfil de variação radial da densidade básica apresentou um padrão de crescimento desde a medula até a casca. Valores de densidade básica foram classificados de médios a baixos, com relação a idade das árvores.

\section{REFERÊNCIAS BIBLIOGRÁFICAS}

AGUIRRE, A. M. et al. Estudo das propriedades físicas e mecânicas da madeira de Podocarpus lambertii Klotzsch oriunda de floresta nativa. In: XVII CIC. X ENPOS, 2008, Pelotas. Anais eletrônicos... Pelotas: UFPe, 2008.

EVANS, J.W.; SENFT, J.F.; GREEN, D.W. Juvenile wood effect in red alder: analysis of physical and mechanical data to delineate juvenile and mature wood zones. Forest Products Journal, Madison, v. 50, n. 7/8, p.75-87, 2000.

FERRAZ, J. S. F.; ALBUQUERQUE, U. P.; MEUNIER, I. M. J. Valor de uso e estrutura da vegetação lenhosa às margens do riacho do Navio, Floresta, PE, Brasil. Acta Botanica Brasílica, v. 20, p. 125-134, 2006.

FPL. Wood Handbook, Wood as an Engineering Material. Forest Products Laboratory. Wood handbook - Wood as an engineering material. General Technical Report FPL-GTR190. Madison, WI: U.S. Department of Agriculture, Forest Service, Forest Products Laboratory, 2010. 508 p. 
LORENZI, H. Árvores brasileiras. Nova Odessa: Plantarum, 2000. v. 1. 352 p.

LORENZI, H. Árvores brasileiras. Nova Odessa: Plantarum, 2009. v. 2.384 p.

MORI, F. A. et al. Utilização de eucaliptos e de madeiras nativas no armazenamento da aguardente de cana-de-açúcar. Ciência e Tecnologia de Alimentos, v.23, p.396-400, 2003.

PAES, J. B. et al. Avaliação do potencial tanífero de seis espécies florestais de ocorrência no semi-árido brasileiro. Cerne, v. 12, p. 232-238, 2006.

PANSHIN, A. J.; DE ZEEUW, C. Textbook of wood technology. McGraw-Hill Book Company. New York. 1970. 722p.

RAMOS et al. Variação radial dos caracteres anatômicos da madeira de Eucalyptus grandis W. Hill Ex Maiden e idade de transição entre lenho juvenil e adulto. Sci. For., Piracicaba, v. 39, n. 92, p. 411-418, dez. 2011

TAMASHIRO, J. Y. Estudos taxonomicos e morfologicos do genero Piptadenia sensu Dentham no sudoeste do Brasil: avaliação das modificações taxonômicas recentemente propostas. 1989. 99 f., Dissertação (Mestrado) - Universidade Estadual de Campinas, Instituto de Biologia.

TEIXEIRA, B. M. R. Variabilidade radial e longitudinal de propriedades da madeira de Anadenathera peregrina (L) Speg. 2008. 72 f., Dissertação (Mestrado em Ciência Florestal) - Universidade Federal de Viçosa.

TRUGILHO, P.F.; SILVA, D.A.; FRAZÃO, F.J.L.; MATOS, J.L.M. Comparação de métodos de determinação da densidade básica em madeira. ACTA Amazônica, Manaus - Amazonas, V. 20, p. 307-319, mar./dez. 1990.

ZOBEL, B.J.; BUIJTENEN, J.P. Wood variation: its causes and control. Berlin: SpringerVerlag, 1989. 361p. 